\title{
A Real-Time Monitor using Wavelet Analysis of the Doppler Heart Sound for the Detection of Venous Air Embolism
}

\author{
Brent C. B. Chan, Student Member, IEEE, Francis H. Y. Chan, Member, IEEE, \\ P. W. Lui, Paul W. F. Poon, F. K. Lam and H. Wang
}

\begin{abstract}
A fast detection algorithm for venous air embolism (VAE) was developed and implemented as a real-time monitor for detecting embolic heart sound and estimating embolic air volume. Its performance was evaluated under bolus injection of sub-clinical ( 0.01 to $0.80 \mathrm{ml})$ and continuous infusion of clinically significant $(0.80$ to $9.60 \mathrm{ml})$ air volumes in anaesthetized dogs. The clinically significant air emboli could be estimated based on the calibration curve obtained during sub-clinical VAE for a subject. The monitor also kept track of the cumulative embolic air volumes and alerted the anaesthetists once a predefined clinically significant embolic air volume was reached. As both humans and dogs share similar physiological conditions, our monitor for dogs are expected to be applicable to humans.
\end{abstract}

\section{INTRODUCTION}

Venous air embolism (VAE), when occurs in large quantity during surgery, is a potential hazard to patients. Precordial Doppler ultrasound is one of the most sensitive and non-invasive monitorings for $\operatorname{VAE}[1,2]$ but it requires the constant attention of anaesthetists to listen to subtle changes in the Doppler heart sound (DHS). Besides, it cannot provide the volume of air entering the heart chamber. Such information is crucial as anaesthetists usually act only when clinically significant embolic air volume is present [3]. Hence, a real-time monitor which can automatically detect the embolic heart sound (EHS) and estimate the embolic air volume is useful for easing the burden of anaesthetists for detecting and assessing the risk of VAE.

Brent C. B. Chan, Francis H. Y. Chan and F. K. Lam are with the Department of Electrical \& Electronic Engineering, The University of Hong Kong, Pokfulam Road, Hong Kong (e-mail: cbchan, fhychan, fklam@eee.hku.hk).

Ping-Wing Lui and Hsin Wang are with the Department of Anesthesiology, Veterans General Hospital-Taipei, Taipei, Taiwan (email: pwlui, hwang@vghtpe.gov.tw).

Paul W. F. Poon is with the Department of Physiology, National Cheng Kung University, 1 University Road, Tainan, Taiwan (e-mail: ppoon@mail.ncku.edu.tw).
In this study, we investigated the DHS under simulated conditions of both sub-clinical (below $1 \mathrm{ml}$ ) and clinically significant (up to $10 \mathrm{ml}$ ) VAE in anaesthetized dogs. With wavelet analysis $[4,5]$, we greatly enhanced the contrast of embolic to normal DHS signal power, facilitating the detection of EHS simply by thresholding. We also developed a fast detection algorithm and implemented it as a real-time monitor for VAE. Based on the calibration curve obtained during sub-clinical VAE, we could estimate the embolic air volume related to each EHS for a subject. Moreover, we could keep track of the cumulative embolic air volumes and alert the anaesthetists when a predefined clinically significant embolic air volume was reached.

\section{THEORY}

\section{A. Wavelet Analysis}

Wavelet analysis is a linear operation which decomposes a signal into components that appear at different scales. The signal is divided into two parts by the wavelet analysis at scale $=1$ based on its spectrum. The low-pass signal (known as the approximation signal) is a coarser approximation of the original signal while the high-pass signal (known as the difference signal) is the difference between them. The same procedure is applied to the approximation signals during wavelet analysis at successive scales. We can compute the approximation signal $A_{2^{*}} f(x)$ and difference signal $D_{2^{*}} f(x)$ of the DHS signal at scale $s$ using the following algorithm [4]:

$$
\begin{aligned}
& A_{2^{s}} f(x)=\sum_{k \in Z} h_{k} A_{2^{s-1}} f\left(x-2^{s-1} k\right) \\
& D_{2^{s}} f(x)=\sum_{k \in Z} g_{k} A_{2^{s-1}} f\left(x-2^{s-1} k\right)
\end{aligned}
$$

where we define, for normalization purpose, the original DHS signal as the approximation signal at scale $=0$, i.e., $A_{2^{\mathrm{o}}} f(x)$. The $h_{k}$ and $g_{k}$ are the impulse responses of the low-pass and high-pass filters, respectively. The 
choice of the wavelet functions only affects the impulse responses and the processing time. In this study, we used the Daubechies 20-coefficient wavelet [5] to analyze the DHS signals. We chose this wavelet as a compromise between its performance and processing time. Further explanation for the wavelet analysis are detailed in other literatures $[4,5]$.

\section{B. Moving Square Average}

Embedding the energy calculation in the moving average [6], we define the moving square average (MSA) of a function $f(x)$ as

$$
\operatorname{MSA}(x)=\frac{1}{L+M+1} \sum_{k=-L}^{M} f^{2}(x-k) .
$$

With a constant scaling of $L+M+1$ (i.e. the window length), (3) can be reduced to

$$
M S A(x)=\sum_{k=-L}^{M} f^{2}(x-k)
$$

It can be shown that the MSA can be computed in a more computationally efficient way as

$$
M S A(x)=\operatorname{MSA}(x-1)+f^{2}\left(x+w_{1}\right)-f^{2}\left(x-w_{2}\right),
$$

where the window length is equal to the sum of $w_{1}$ and $w_{2}$. The MSA is useful for detecting EHS.

\section{EXPERIMENT}

The study was approved by the Animal Research Committee of the Veterans General Hospital-Taipei, Taiwan. Four mongrel dogs, weighing $10-15 \mathrm{~kg}$, were anaesthetized and placed in the supine position with their lungs being mechanically ventilated. To simulate the sub-clinical and clinically significant VAE, we performed a series of bolus injection of small air volumes $(0.01,0.02,0.05,0.07,0.10,0.15,0.20,0.30$, 0.40 and $0.80 \mathrm{ml}$ ), followed by continuous infusion of larger volumes $(0.80,1.60,2.40,4.80$ and $9.60 \mathrm{ml})$ at various controlled rates of $0.011-0.192 \mathrm{ml} \mathrm{kg}^{-1} \mathrm{~min}^{-1}$ over 5 minutes via the right external jugular vein. Preinjection baseline period ( 5 minutes) and intermission in between successive infusions ( 2 to 5 minutes) were allowed. The DHS during the entire experiment was recorded on a tape recorder, which acted later as the simulation input for the real-time monitor.

\section{ALGORITHM}

The wavelet analysis of the DHS at scale $=1$ greatly enhances the contrast of embolic to normal DHS signal power. The MSA of the wavelet-processed DHS shows this enhancement (Fig. 1). Detecting EHS can thus be facilitated by thresholding on the MSA. The threshold is set to be two times the mean peak of MSA of the past 1- minute wavelet-processed DHS signals on empirical grounds. Essentially, an adaptive thresholding is established which adapts to the possible change of magnitude of the DHS signals due to prolonged operation. In addition, the cumulative embolic energy is found to be linearly (log-log scale) related to the volume of air both from the bolus and continuous infusions. Hence, the clinically significant embolic air volume can be estimated based on the calibration curve obtained during sub-clinical VAE. The cumulative embolic air is monitored and alarming signal is issued once a predefined clinically significant embolic air volume is reached.

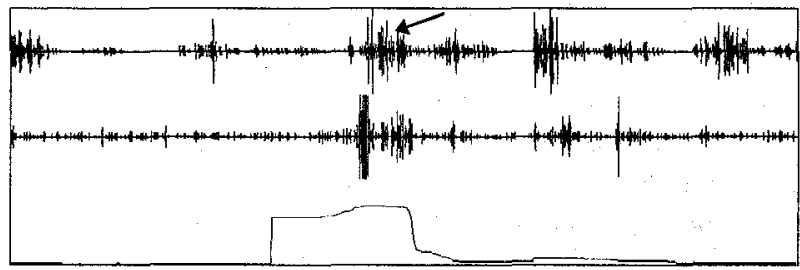

Fig. 1. The DHS signal (upper tracing), the wavelet analysis of the DHS at scale $=1$ (middle tracing) and its MSA (bottom tracing). EHS after bolus air injection of $0.01 \mathrm{ml}$ is marked by arrow.

\section{REAL-TIME MONITOR}

Our real-time monitor for $\mathrm{VAE}$ is a software package developed using the Borland $\mathrm{C}++$ language. It runs under the environment of Microsoft Windows 3.1 or Windows 95 . We installed our real-time monitor in a PC (100 MHz Pentium with $16 \mathrm{MB}$ RAM) for testing and simulation. A 16-bit sound card is required for importing and analogue-to-digital (A/D) converting the audio DHS. The real-time monitoring of the continuous DHS is facilitated by partitioning it into a series of windowed DHS. With the help of the Windows multimedia extensions, we can start the $\mathrm{A} / \mathrm{D}$ conversion (at $5.5 \mathrm{kHz}$ ) of each windowed DHS in the background and process the previously $\mathrm{A} / \mathrm{D}$ converted windowed DHS at the same time.

The DHS signals entering the monitor are processed by three main procedures, namely wavelet analysis at scale $=1$, MSA of the wavelet-processed DHS and location of the peak of MSA. The tracings and various parameters are display in the panel of the real-time monitor (Fig. 2). In the upper half of the panel shows, from top to bottom, the original DHS signal, the wavelet analysis of the DHS at scale $=1$ and the MSA of the middle tracing. The monitor is designed to be operated in three modes which are detailed in the lower half of the panel. In the learning mode, the control baseline period of the DHS was fed to the monitor for calculating the mean energy of the wavelet-processed DHS and the mean peak of the MSA for normalization purpose in 
subsequent modes. Then, the DHS with bolus injection of small air volumes entered the monitor in the calibration mode so as to establish a calibration curve. Finally, the DHS with continuous infusion of larger air volumes was fed to the monitor in the monitoring mode. The embolic energy of the EHS is fitted to the calibration curve for estimating embolic air volume. In the lower right region of the panel shows the real-time trace of the cumulative embolic air volume (y-axis) against the infusion time ( $x$-axis). The trace is cleared every 10 minutes in order to accommodate later traces and all the traces can be stored for future reference. This real-time trace is important and useful for anaesthetists to insure proper clinical treatments during surgery.

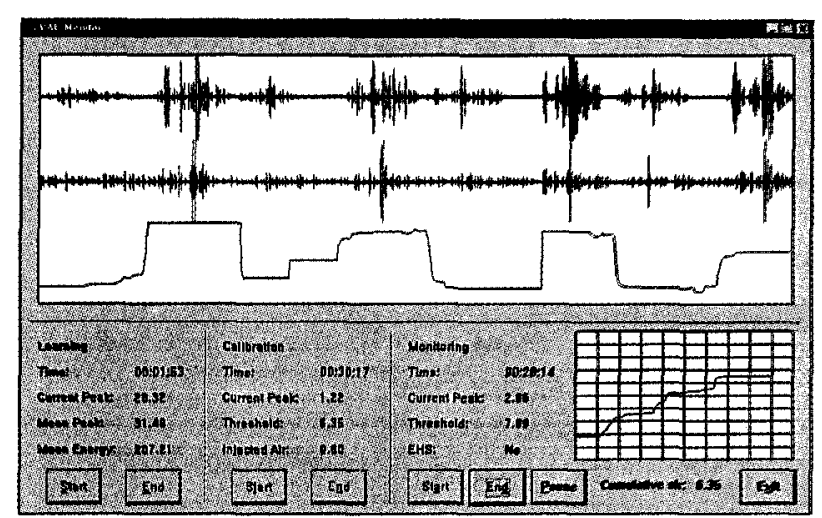

Fig. 2. The panel of the real-time monitor. For the real-time trace in the lower right region of the panel, the infusion time ( $\mathrm{x}$-axis) is from 0 to 10 minutes while the cumulative embolic air volume ( $y$-axis) is from 0 to $10 \mathrm{ml}$

\section{RESULTS}

The linear regression results of the four dogs during bolus and continuous infusions are summarized in Table I. Except for the intercepts of Dog A, there was statistically no difference $(\mathrm{P}<0.05$ using Student's t-test [7]) in the slopes and intercepts between the regression lines for both cases, reassuring that the clinically significant embolic air volume can be estimated based on the calibration curve obtained during sub-clinical VAE. Fig. 3 shows a typical plot of the cumulative embolic air volume against the infusion time, showing the gradual build-up of the embolic air volumes during infusion. The cumulative embolic air volume increased irregularly during the infusion period while it followed a linear trend in the intermission.

\section{DISCUSSION AND CONCLUSION}

We observed remarkable similarity in the EHS between the bolus injection and continuous infusion. The EHS due to continuous infusion was similar to that during a series of bolus injection. In fact, this was the rationale in this study for estimating clinically significant embolic air volumes based on the calibration curve obtained during sub-clinical VAE. Although the rate of continuous infusion was kept constant for each infusion, the EHS with different embolic energy occurred in an intermittent manner. The irregular increase of the cumulative embolic air volume in Fig. 3 showed this fact. This phenomenon may probably reflect the non-linearity of the air emboli travelling from the external jugular vein to the right atrium. In the intermission, some traces of EHS were also detected and the cumulative embolic air volume was observed to increase linearly (Fig. 3), which may probably due to the re-circulation of the air emboli within the heart chamber. This linear increase, if persists after surgery, may indicate that embolic air is still resident and suitable clinical treatments have to be done. Although our simulation experiment was carried out on dogs, similar results for humans are expected as they would share similar physiological conditions. As the heart rate of dogs is higher than that of adult humans, the feasibility of real-time monitoring of VAE in dogs also suggests the same results for humans.

\begin{tabular}{|c|c|c|c|c|c|c|c|c|}
\hline \multirow[b]{2}{*}{ Dog } & \multicolumn{4}{|c|}{ Bolus Injection } & \multicolumn{4}{|c|}{ Continuous Infusion } \\
\hline & Slope & Intercept & r & Pe & Slope & intercept & $r$ & $\mathrm{P}<$ \\
\hline$A$ & 1.82 & 12.77 & 0.93 & 0.001 & 1.25 & 10.59 & 0.93 & 0.05 \\
\hline B & 0.74 & 7.23 & 0.64 & 0.05 & 1.47 & 6.77 & 0.96 & 0.025 \\
\hline c & 0.83 & 4.61 & 0.93 & 0.001 & 0.47 & 5.13 & 0.79 & ns \\
\hline D & 1.22 & 10.34 & 0.91 & 0.001 & 0.41 & 10.98 & 0.65 & ns \\
\hline
\end{tabular}

Table I. The regression statistics of the bolus injection and continuous infusion data. For each dog, no statistical difference (except the intercepts of Dog A) exists in the slopes and intercepts of the regression line $(P<0.05)$ between bolus injection and continuous infusion. Abbreviations: $\mathrm{r}=$ correlation coefficient; $\mathrm{ns}=$ not significant (i.e. $P>0.05$ ).

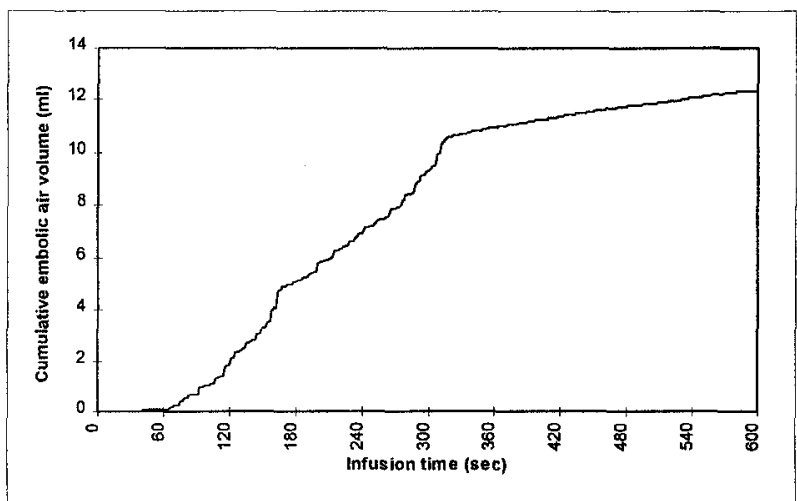

Fig. 3. Plot of the estimated cumulative embolic air volume against the infusion time of $9.60 \mathrm{ml}$ air for Dog A. Air infusion during the first half ( 0 to 300 seconds) while intermission in the second half (300 to 600 seconds).

One of the favourable features of our real-time monitor is the ability to estimate embolic air volumes. 
During the continuous air infusion, the volume of each air emboli related to each EHS can be estimated based on the previously established calibration curve. The cumulative embolic air volume can thus be obtained by summing all the previous volume of air emboli. This cumulative embolic air volume is particular useful as it can be used to alert the anaesthetists at a predefined clinically significant embolic air volume (e.g. $1 \mathrm{ml}$ ) so that clinical treatments such as retrieval of air emboli using catheter can be performed without delay.

Another features of the real-time monitor is the adaptive thresholding which was firstly proposed in [8]. This thresholding technique acquires constantly the peaks of MSA of the previous wavelet-processed windowed DHS and computes the appropriate threshold for detecting EHS. This adaptive technique is more favourable than a fixed threshold in that reliable and robust detection can be achieved while false alarm is kept to a minimum. Besides, it can adapt to the drift of magnitude of the DHS in case the position of the ultrasonic probe is altered or some parameters of the surgical instruments are changed due to prolonged operation.

Though the DHS has a periodic rhythm $(0.25 \mathrm{~Hz})$ in its magnitude as a result of the chest wall movement produced by the ventilator, our wavelet method can still detect the EHS. Its sensitivity is $0.01 \mathrm{ml}$ and the detection range is up to almost $10 \mathrm{ml}$, sufficient to cover both the sub-clinical and clinically significant embolic air volumes.

A real-time monitor for VAE was realized using the wavelet analysis of the DHS at scale $=1$ and its MSA as the main algorithms. The real-time implementation is facilitated by windowing the continuous DHS, A/D converting the windowed DHS in the background and processing the previously $\mathrm{A} / \mathrm{D}$ converted windowed DHS using our algorithms. The clinically significant air emboli can be estimated based on the calibration curve obtained during sub-clinical VAE for a subject. The monitor can also keep track of the cumulative embolic air volumes and alerts the anaesthetists once a predefined clinically significant embolic air volume is reached.

\section{ACKNOWLEDGMENT}

This work was supported in part by The University of Hong Kong Research Grants and National Science Council NSC 85-2331-B075-008 (PWL), Taiwan.

\section{REFERENCES}

[1] R. W. Buckland and I. M. Manners, "Venous air embolism during neurosurgery: a comparison of various methods of detection in man," Anaesthesia, vol. 31, pp. 633-643, 1976.

[2] J. B. English, D. Westenskow, M. R. Hodges and T. H. Stanley, "Comparison of venous air embolism monitoring methods in supine dogs," Anesthesiology, vol. 48, pp. 425-429, 1978.

[3] R. C. Shupak, "Air embolism and its influence on anesthetic management," Current Reviews in Clinical Anesthesia, vol. 11, pp. 113-124, 1991.

[4] S. G. Mallet, "A theory of multiresolution signal decomposition: the wavelet representation," IEEE Transactions on Pattern Analysis and Machine Intelligence, vol. 11, pp. 674-693, 1989.

[5] I. Daubechies, "Ten lectures on wavelets," Philadelphia, Pennsylvania, Society for Industrial and Applied Mathematics, 1992.

[6] A. V. Oppenheim and R. W. Schafer, "Discretetime signal processing," Englewood Cliffs, NJ, Prentice-Hall, 1989.

[7] H. L. Alder and E. B. Roessler, "Introduction to probability and statistics," San Francisco, California, W. H. Freeman and Company, 1972.

[8] B. C. B. Chan, F. H. Y. Chan, F. K. Lam, P. W. Lui and P. W. F. Poon, "Real-time monitoring of venous air embolism with Doppler heart sound using multiresolution wavelet analysis," Engineering and Physics in Medicine, Queenstown, New Zealand, Conference Programme, p. 185, Nov. 1995. 